\title{
The environment of the infrared dust bubble N65: a multiwavelength study
}

\author{
A. Petriella, S. Paron, and E. Giacani \\ Instituto de Astronomía y Física del Espacio (CONICET-UBA), CC 67, Suc. 28, 1428 Buenos Aires, Argentina \\ e-mail: apetriella@iafe.uba.ar
}

Received 6 November 2009 / Accepted 14 January 2010

\begin{abstract}
Aims. We investigate the environment of the infrared dust bubble N65 and search for evidence of triggered star formation in its surroundings.

Methods. We performed a multiwavelength study of the region around N65 with data taken from large-scale surveys: Two Micron All Sky Survey, GLIMPSE, MIPSGAL, SCUBA, and GRS. We analyzed the distribution of the molecular gas and dust in the environment of N65 and performed infrared photometry and spectral analysis of point sources to search for young stellar objects and identify the ionizing star candidates.

Results. We found a molecular cloud that appears to be fragmented into smaller clumps along the N65 PDR. This indicates that the so-called collect and collapse process may be occurring. Several young stellar objects are distributed among the molecular clumps. They may represent a second generation of stars whose formation was triggered by the bubble expanding into the molecular gas. We identified O-type stars inside N65, which are the most reliable ionizing star candidates.
\end{abstract}

Key words. HII regions - ISM: clouds - stars: formation

\section{Introduction}

Since the early work of Elmegreen \& Lada (1977), it is known that during the supersonic expansion of an HII region, a dense layer of material can be collected between the ionization and the shock fronts. This layer can be fragmented into massive condensations that may then collapse to form new massive stars and/or clusters. Several observational studies have inferred that this process, known as "collect and collapse", triggers massive star formation (see e.g., Pomarès et al. 2009; Zavagno et al. 2007, and references therein). As Deharveng et al. (2005) point out, only the presence of either a dense molecular shell surrounding the ionized gas of an HII region or massive fragments regularly spaced along the ionization front, can prove that we are dealing with the collect and collapse process.

Using Galactic Legacy Infrared Mid-Plane Survey Extraordinaire (GLIMPSE) data, Churchwell et al. (2006, 2007) cataloged almost 600 infrared (IR) dust bubbles, full or partial rings bordered by a photodissociation region (PDR) and detected mainly at $8 \mu \mathrm{m}$, which usually enclose ionized gas and hot dust observed at $24 \mu \mathrm{m}$. Most of these bubbles are HII regions and several have morphologies that are indicative of triggered star formation.

On the other hand, Cyganowski et al. (2008) also using GLIMPSE data, identified more than 300 extended $4.5 \mu \mathrm{m}$ sources, so-called "extended green objects (EGOs)", applying the common coding of the [4.5] band as green in three-color composite Infrared Array Camera images. According to the authors, an EGO is probably a massive young stellar object (MYSO) driving outflows. The extended emission in the $4.5 \mu \mathrm{m}$ band is produced by $\mathrm{H}_{2}(v=0-0, \mathrm{~S}(9,10,11))$ lines and $\mathrm{CO}(v=$ $1-0$ ) band heads, which are excited by the shock of the outflows propagating in the interstellar medium (ISM). The majority of EGOs are associated with infrared dark clouds (IRDCs) and some lie over the border of IR dust bubbles.

From the catalog of Churchwell et al. (2006), we select the IR dust bubble N65, which harbors the EGO G35.03+0.35 in one of its borders (indicative of active star formation), to study its environment.

N65 is a complete (i.e., closed ring) IR dust bubble centered on $\alpha_{2000}=18^{\mathrm{h}} 54^{\mathrm{m}} 02^{\mathrm{s}}, \delta_{2000}=+01^{\circ} 59^{\prime} 30^{\prime \prime}\left(l=35^{\circ} .000\right.$, $b=+0^{\circ} 332$ ) with a radius of about $2^{\prime} .6$ (Churchwell et al. 2006). Close to N65, over one of its borders, centered on $\alpha_{2000}=$ $18^{\mathrm{h}} 54^{\mathrm{m}} 04.2^{\mathrm{s}}, \delta_{2000}=+02^{\circ} 01^{\prime} 33.9^{\prime \prime}\left(l=35^{\circ} .035, b=+0^{\circ} .338\right)$ lies the IR source IRAS $18515+0157$, where several molecular lines tracers of star formation were detected. Maser emission from $\mathrm{H}_{2} \mathrm{O}$ and $\mathrm{OH}$ was detected by Forster \& Caswell (1989), as well as methanol (Caswell et al. 1995). Bronfman et al. (1996) and Jijina et al. (1999) also detected CS and $\mathrm{NH}_{3}$, respectively. The detection of formaldehyde at $v_{\mathrm{LSR}} \sim 57 \mathrm{~km} \mathrm{~s}^{-1}$ was used by Watson et al. (2003) to determine that this star-forming region lies at the kinematic distance of $10 \mathrm{kpc}$, the farthest possible given the distance ambiguity of the first Galactic quadrant. On the other hand, close to this region, at $l=35^{\circ} .015$, $b=+0.356$ and also across the border of N65, Anderson et al. (2009) cataloged the UCHII region G35.02+0.35 with a velocity of $\sim 57.2 \mathrm{~km} \mathrm{~s}^{-1}$, which they locate at the near distance of $3.6 \mathrm{kpc}$ (Anderson \& Bania 2009).

In this work, we present a molecular and IR study of the environment surrounding the IR dust bubble N65 to explore its surrounding ISM and search for signatures of star formation. We describe the data used in Sect. 2, the results and the discussion of them are presented in Sect. 3 and Sect. 4 summarizes the results. 

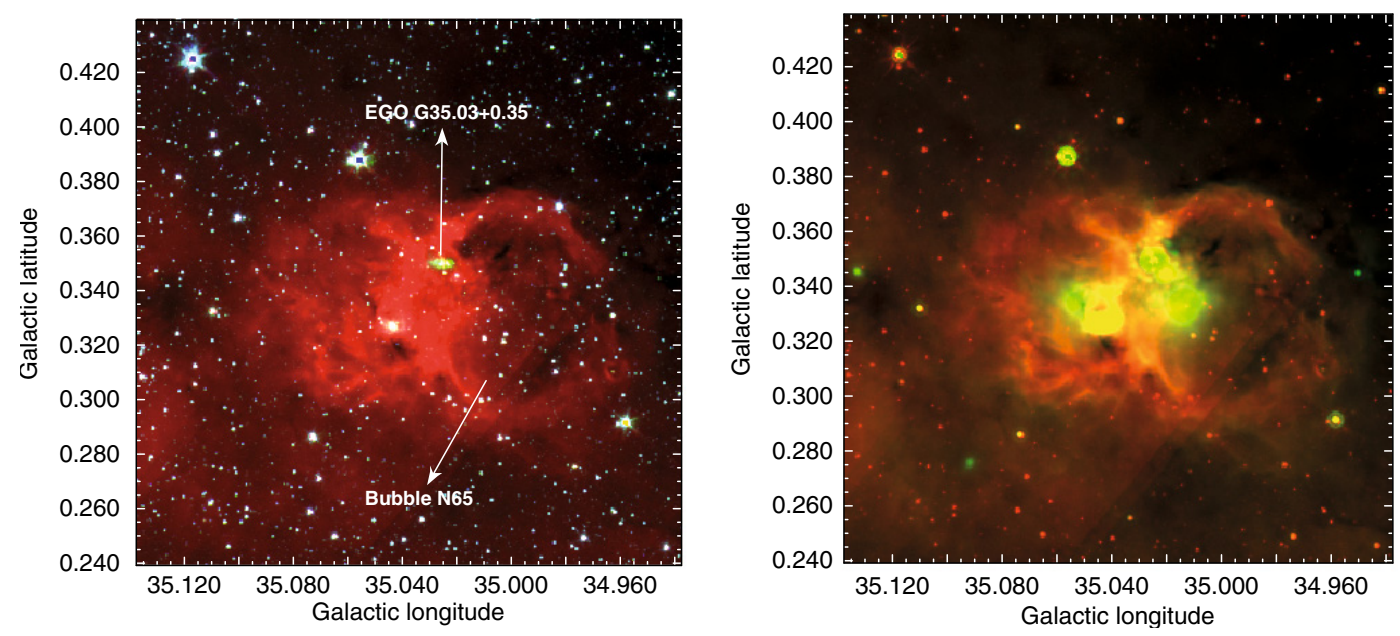

Fig. 1. Mid-IR emission of the IR dust bubble N65. Left: Spitzer-IRAC three-color image $(3.6 \mu \mathrm{m}=$ blue, $4.5 \mu \mathrm{m}=$ green, and $8 \mu \mathrm{m}=$ red). The location of the EGO G35.03+0.35 is indicated. Right: red is the Spitzer-IRAC $8 \mu \mathrm{m}$ emission and green is the Spitzer-MIPSGAL emission at $24 \mu \mathrm{m}$.

\section{Data}

We analyzed data extracted from five large-scale surveys: the Two Micron All Sky Survey $(2 \mathrm{MASS})^{1}$, Galactic Legacy Infrared Mid-Plane Survey Extraordinaire (GLIMPSE), MIPSGAL, GRS ${ }^{2}$, and the SCUBA Legacy Catalogue. GLIMPSE is a mid-infrared survey of the inner Galaxy performed with the Spitzer Space Telescope. We used the mosaicked images from GLIMPSE and the GLIMPSE Point-Source Catalog (GPSC) in the Spitzer-IRAC (3.6, 4.5, 5.8, and $8 \mu \mathrm{m})$. IRAC has an angular resolution of between 1".5 and 1".9 (see Fazio et al. 2004; and Werner et al. 2004). MIPSGAL is a survey of the same region as GLIMPSE, using the MIPS instrument $(24$ and $70 \mu \mathrm{m})$ on Spitzer. The MIPSGAL resolution is $6^{\prime \prime}$ at $24 \mu \mathrm{m}$. The GRS is being performed by the Boston University and the Five College Radio Astronomy Observatory (FCRAO). The survey maps the galactic Ring in the ${ }^{13} \mathrm{CO} J=$ $1-0$ line with an angular and spectral resolution of $46^{\prime \prime}$ and $0.2 \mathrm{~km} \mathrm{~s}^{-1}$, respectively (see Jackson et al. 2006). The observations were performed in both position-switching and on-thefly mapping modes, achieving an angular sampling of $22^{\prime \prime}$. The SCUBA Legacy Catalogues ${ }^{3}$ are two sets of continuum maps and catalogs using data at 450 and $850 \mu \mathrm{m}$ obtained with the Submillimetre Common User Bolometer Array (SCUBA) with angular resolutions of $19^{\prime \prime}$ and $11^{\prime \prime}$, respectively (Di Francesco et al. 2008).

\section{Results and discussion}

Figure 1 (left) shows a Spitzer-IRAC three color image of N65. The three IR bands are $3.6 \mu \mathrm{m}$ (in blue), $4.5 \mu \mathrm{m}$ (in green), and $8 \mu \mathrm{m}$ (in red). The location of the EGO G35.03+0.35 (Cyganowski et al. 2008) is indicated. Figure 1 (right) displays a composite two-color image towards N65. Red and green represent the Spitzer-IRAC emission at $8 \mu \mathrm{m}$ and the SpitzerMIPSGAL emission at $24 \mu \mathrm{m}$, respectively. Both figures clearly

1 2MASS is a joint project of the University of Massachusetts and the Infrared Processing and Analysis Center/California Institute of Technology, funded by the National Aeronautics and Space Administration and the National Science Foundation.

2 Galactic Ring Survey (Jackson et al. 2006).

${ }^{3}$ http://www2 . cadc-ccda.hia-iha.nrc-cnrc.gc.ca/ community/scubalegacy/
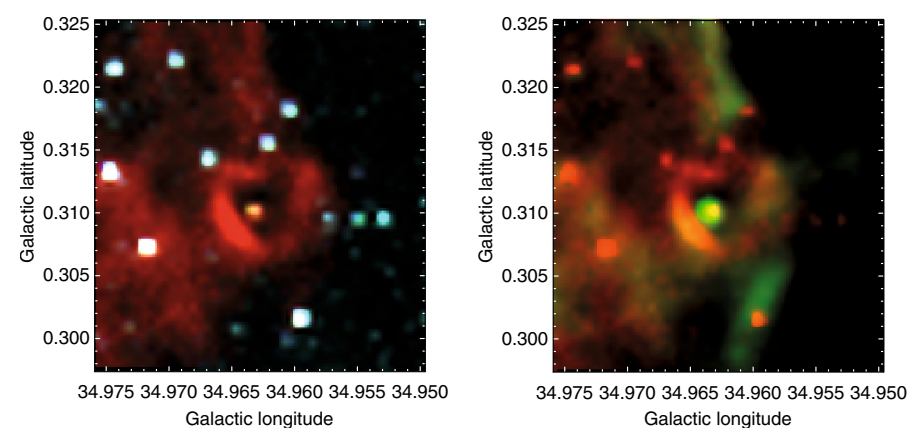

Fig. 2. Mid-IR emission of the possible smaller IR dust bubble that lies in the southwest border of N65. Left: Spitzer-IRAC three-color image ( $3.6 \mu \mathrm{m}=$ blue, $4.5 \mu \mathrm{m}=$ green and $8 \mu \mathrm{m}=$ red). Right: red is the Spitzer-IRAC $8 \mu \mathrm{m}$ emission and green is the Spitzer-MIPSGAL emission at $24 \mu \mathrm{m}$.

show the PDR visible in the $8 \mu \mathrm{m}$ emission, which originates mainly in the polycyclic aromatic hydrocarbons (PAHs). The PAH emission delineates the HII region boundaries because these large molecules are destroyed inside the ionized region, but are excited in the PDR by the radiation leaking from the HII region (Pomarès et al. 2009). The $24 \mu \mathrm{m}$ emission, displayed in green in Fig. 1 (right), corresponds to hot dust, distributed mainly towards the eastern border of N65 that coincides with the central position of IRAS $18515+0157$.

From Fig. 1, a probably smaller IR dust bubble can be discerned in the southwest border of N65, centered on $l=34.96$, $b=+0.31$, which is displayed in Fig. 2. The emission at $8 \mu \mathrm{m}$ (Fig. 2 left) shows an almost circular PDR, which encloses a region of about $18^{\prime \prime}$ in diameter. At the center of the bubble, a source is visible with emission in the $3.5,4.5,8$, and $24 \mu \mathrm{m}$ bands, which is probably the exciting star of this structure surrounded by hot dust emitting at $24 \mu \mathrm{m}$ (green in Fig. 2 right).

\subsection{Molecular and dust analysis}

We inspected the molecular gas around N65 from the GRS data in the whole velocity range and found an interesting feature around $v \sim 50 \mathrm{~km} \mathrm{~s}^{-1}$. Figure 3 shows the integrated velocity maps of the ${ }^{13} \mathrm{CO} J=1-0$ emission every $1.05 \mathrm{~km} \mathrm{~s}^{-1}$ between 47 and $56 \mathrm{~km} \mathrm{~s}^{-1}$. The circle shows the position and size 


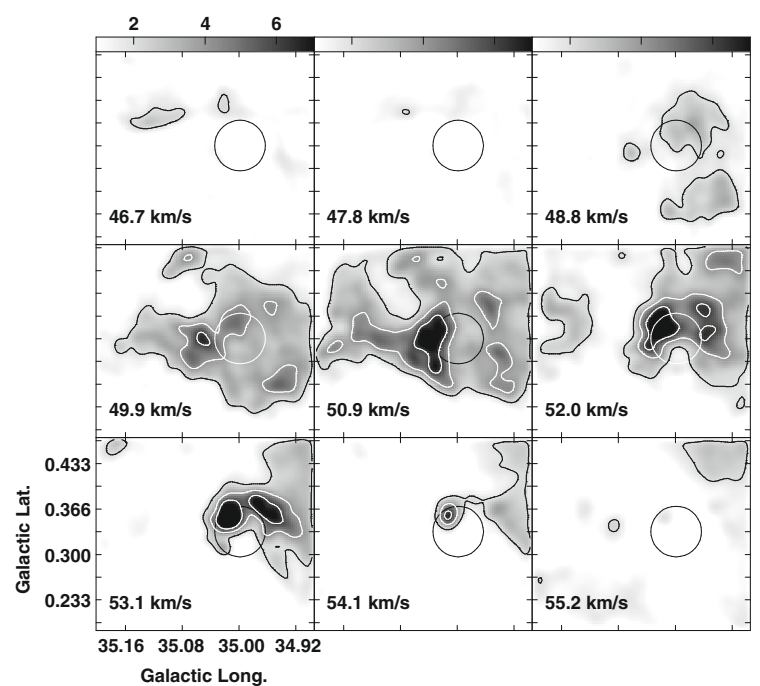

Fig. 3. Integrated velocity maps of the ${ }^{13} \mathrm{CO} J=1-0$ emission every $1.05 \mathrm{~km} \mathrm{~s}^{-1}$. The grey scale is in $\mathrm{K} \mathrm{km} \mathrm{s}^{-1}$. The contour levels are 2 , 4 and $6 \mathrm{~K} \mathrm{~km} \mathrm{~s}^{-1}$. The circle represents the position and size of N65.

of N65. A molecular cloud with an arc-like shape open to the south is evident that encloses the IR bubble over one of its borders. The molecular gas exhibits several clumps along the PDR. The distribution and morphology of this material suggests that the collect and collapse process might be occurring.

In Fig. 4, we compare the ${ }^{13} \mathrm{CO}$ emission integrated within the interval 47 and $55 \mathrm{~km} \mathrm{~s}^{-1}$ with the continuum dust emission at $850 \mu \mathrm{m}$ extracted from the SCUBA (in yellow contours). In this figure, it is evident that the dust emission coincides with the brightest molecular clump centered on $l \sim 35^{\circ} .02, b \sim 0.35$ and with the position of the EGO G35.03+0.35. The presence of the EGO together with all the previous results mentioned in Sect. 1 provide clear evidence that this region might be active in star formation.

If we assume that the molecular gas in the velocity range considered before is physically associated with N65, we can adopt $\sim 50 \mathrm{~km} \mathrm{~s}^{-1}$ as the systemic velocity of the infrared dust bubble. According to the galactic rotation model of Fich et al. (1989) (with $R_{\odot}=8.5 \mathrm{kpc}$ and $v_{\odot}=220 \mathrm{~km} \mathrm{~s}^{-1}$ ), we obtain kinematic distances of either 3.5 or $10.5 \mathrm{kpc}$. Considering that the detection rate for IR dust bubbles in GLIMPSE peaks at the distance of $4.2 \mathrm{kpc}$ within a horizon of $8 \mathrm{kpc}$ (Churchwell et al. 2006), the bubbles at the near kinematic distance are more likely to be detected than those at the far distance. Thus, we assume a distance of $3.5 \mathrm{kpc}$ for N65, in coincidence with the UCHII region G35.02+0.35 cataloged by Anderson \& Bania (2009).

Using the ${ }^{13} \mathrm{CO} J=1-0$ line and assuming local thermodynamic equilibrium (LTE), we estimate the $\mathrm{H}_{2}$ column density toward the brightest molecular clump shown in Fig. 4. We use

$N\left({ }^{13} \mathrm{CO}\right)=2.42 \times 10^{14} \frac{T_{\mathrm{ex}} \int \tau_{13} \mathrm{~d} v}{1-\exp \left(-5.29 / T_{\mathrm{ex}}\right)}$,

to obtain the ${ }^{13} \mathrm{CO}$ column density, where $\tau_{13}$ is the optical depth of the line and following Anderson et al. (2009) we considered $T_{\text {ex }}=20 \mathrm{~K}$. We assume that the ${ }^{13} \mathrm{CO}$ emission is optically thin and use the relation $N\left(\mathrm{H}_{2}\right) / N\left({ }^{13} \mathrm{CO}\right) \sim 5 \times 10^{5}$ (e.g., Simon et al. $2001)$ to estimate a column density of $N\left(\mathrm{H}_{2}\right) \sim 1.6 \times 10^{22} \mathrm{~cm}^{-2}$. From this value, we estimate the mass of the molecular clump to be $\sim 2 \times 10^{3} M_{\odot}$. This value was obtained from

$M=\mu m_{\mathrm{H}} \sum\left[D^{2} \Omega N\left(\mathrm{H}_{2}\right)\right]$,

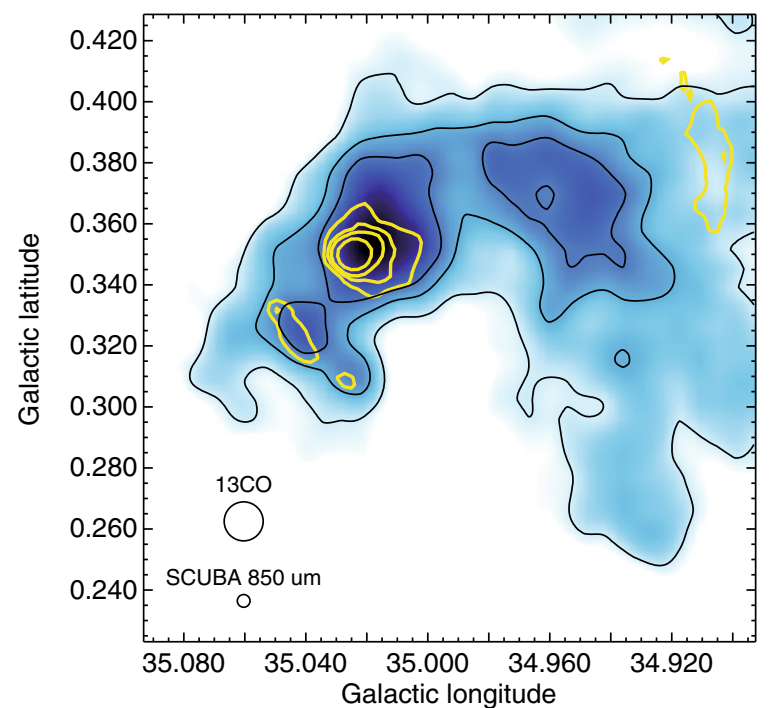

Fig. 4. ${ }^{13} \mathrm{CO}$ emission integrated between 47 and $55 \mathrm{~km} \mathrm{~s}^{-1}$, the contours levels are 19,23 , and $33 \mathrm{~K} \mathrm{~km} \mathrm{~s}^{-1}$. The yellow contours correspond to the $850 \mu \mathrm{m}$ continuum emission obtained from SCUBA and the levels are $0.3,0.6,0.9$, and $2.3 \mathrm{Jy} \mathrm{beam}^{-1}$. The beams of each emission are included in the bottom left corner.

where $\Omega$ is the solid angle subtended by the ${ }^{13} \mathrm{CO} J=1-0$ beam size, $m_{\mathrm{H}}$ is the hydrogen mass, $\mu$ is the mean molecular weight assumed to be 2.8 by taking into account a relative helium abundance of $25 \%$, and $D$ is the distance. Summation was performed over all the observed positions within the $33 \mathrm{~K} \mathrm{~km} \mathrm{~s}^{-1}$ contour level (see Fig. 4). Finally from this mass value, we derive a density of $n \sim 10^{4} \mathrm{~cm}^{-3}$.

Using the $850 \mu \mathrm{m}$ continuum emission, we estimate the dust mass from the relation of Tej et al. (2006):

$M_{\text {dust }}=1.88 \times 10^{-4}\left(\frac{1200}{v}\right)^{3+\beta} S_{v}\left(\mathrm{e}^{0.048 v / T_{\mathrm{d}}}-1\right) D^{2}$,

where $S_{v}$ is the flux density at the frequency $v$. We use $S_{353 \mathrm{GHz}}=19.42 \mathrm{Jy}$ as obtained by Di Francesco et al. (2008) for this region. We assume that $T_{\mathrm{d}}$ the dust temperature is $20 \mathrm{~K}$, $\beta$ the dust emissivity index is 2.6 for the assumed dust temperature in this region according to Hill et al. (2006), and $D$ the distance to N65 is $3.5 \mathrm{kpc}$. This equation assumes a standard opacity of $k_{1200 \mathrm{GHz}}=0.1 \mathrm{~cm}^{2} \mathrm{~g}^{-1}$. We obtain a dust mass of $M_{\text {dust }} \sim 60 M_{\odot}$. The dust-to-gas mass ratio is then $\sim 0.03$, which is 3 times higher than the canonical Galactic ratio.

\subsection{IR photometry}

We analyze the distribution of IR point sources in the surroundings of N65 to search for signs of star formation and try to identify the ionizing-star candidates. Figure 5 shows the [5.8]-[8.0] versus [3.6]-[4.5] color-color (CC) diagram for the sources extracted from the GLIMPSE Point Source Catalog in the SpitzerIRAC bands within a circle of $8^{\prime}$ in radius centered on $l=35^{\circ} .00$, $b=+0.32$. The size of this region completely covers the extension of N65 and the surrounding molecular clumps, where stars may be forming. We only consider sources with detection in the four bands. The regions in the figure indicate the stellar evolutionary stages based on the criteria of Allen et al. (2004): class I sources are protostars with circumstellar envelopes, class II are disk-dominated objects, and class III are main sequence and giant stars. We search for ionizing star candidates among class III 


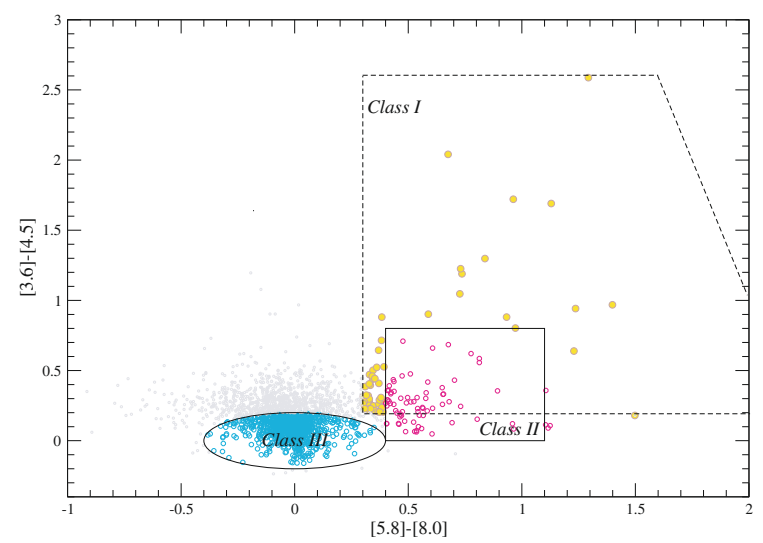

Fig. 5. GLIMPSE color-color diagram [5.8]-[8.0] versus [3.6]-[4.5] for sources within a circle of $8^{\prime}$ in radius centered at N65. We only consider sources with detection in the four Spitzer-IRAC bands. The regions indicate the stellar evolutionary stage as defined by Allen et al. (2004). Class I sources are protostar with circumstellar envelopes; class II are disk dominated objects; and class III are main sequence and giant stars.

sources, while class I and II sources are chosen to be the YSO candidates. In the following sections, we report the results of these analyses.

\subsection{Identifying the exciting star(s) of N65}

We attempt identify or at least propose the possible exciting stars(s) of N65.

According to Churchwell et al. (2006), IR dust bubbles may be produced by $\mathrm{O}-$ and/or B-type stars. Given that we do not find any cataloged massive star toward N65, we look for the exciting star(s) among the class III sources because they include main sequence stars. The ionizing star(s) are not always located close to the geometrical center of the bubble because of their proper motion. However, we expect to find them inside the PDR as IR dust bubbles are young objects and the ionizing star(s) may not have enough time to overcome the shell. To perform near IR photometry using the 2MASS JHK bands, we select class III objects within the N65 PDR that have detections in the aforementioned near IR bands. Figure 6 shows the $(H-K)$ versus $(J-H)$ color-color (CC) diagram of the 22 sources found using this criteria. Figure 7 shows the $K$ versus $(H-K)$ color-magnitude (CM) diagram. Finally, in Fig. 8 we show the location of these sources.

To identify possible exciting star(s) candidates among the 22 sources found, we select objects that meet both of the following criteria simultaneously: (i) according to their position in the CC diagram (Fig. 6), the sources may be main sequence stars and (ii) according to their position in the CM diagram (Fig. 7), the sources may be O- or B-type stars. These sources are indicated by red numbers in Figs. 6 and 7 and red crosses in Fig. 8.

As an additional constraint of the ionizing star(s) candidate, we derive the spectral energy distribution (SED) of the selected sources by fitting the fluxes in the IRAC and 2MASS bands with a Kurucz photospheric model using the tool developed by Robitaille et al. (2007) and available online ${ }^{4}$. The fitting tool requires a distance range and a range of visual extinction. We assume a distance of $3.5 \mathrm{kpc}$ and derive the extinction for each source from the 2MASS CC diagram $(H-K)$ versus $(J-H)$ presented in Fig. 6. Once the SED is obtained, we check whether the

\footnotetext{
${ }^{4}$ http://caravan.astro.wisc.edu/protostars/
}

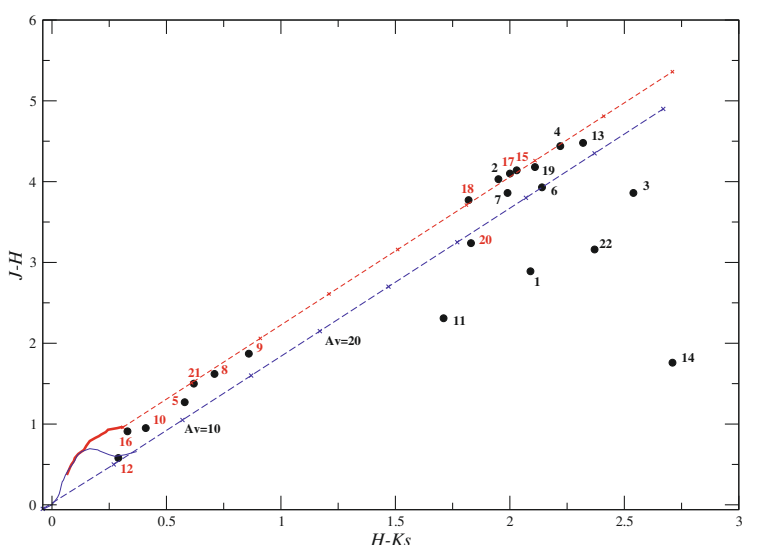

Fig. 6. $(H-K)$ versus $(J-H)$ color-color $(\mathrm{CC})$ diagram of the class III objects within the N65 PDR. The two solid curves represent the location of the main sequence (thin line) and the giant stars (thicker line) derived from Bessell \& Brett (1988). The parallel dashed lines are reddening vectors. We have assumed the interstellar reddening law of Rieke \& Lebofsky (1985) $\left(A_{J} / A_{V}=0.282 ; A_{H} / A_{V}=0.175\right.$ and $\left.A_{K} / A_{V}=0.112\right)$. The sources with red numbers are the ones selected to be the candidates for exciting stars of N65.

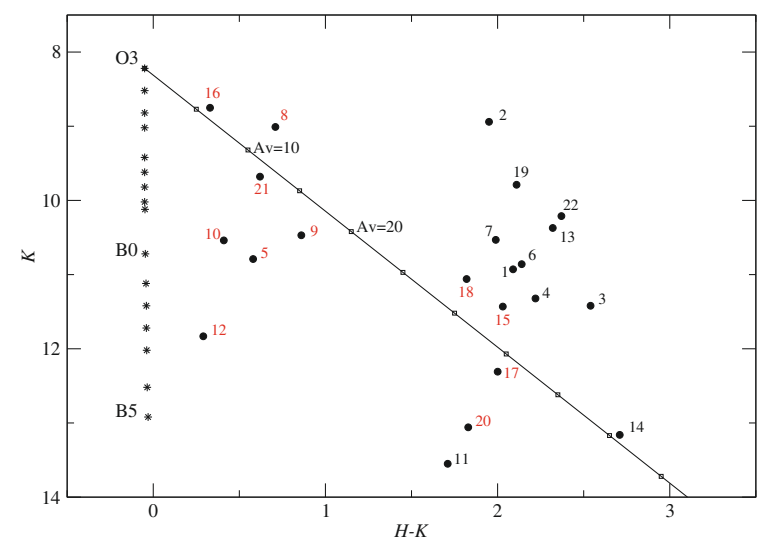

Fig. 7. $(H-K)$ versus $(K)$ color-magnitude $(\mathrm{CM})$ diagram of the class III objects within the N65 PDR. The position of the zero-age-mainsequence stars with $A_{v}=0$ mag at a distance of $3.5 \mathrm{kpc}$ are indicated by black stars. The reddening curve for an $\mathrm{O} 3$ star is shown with a black line. The sources with red numbers represent the ones selected to be candidate exciting stars of N65.

fitted effective temperature is consistent with the temperature of an O- and B-type star. Our analysis is only qualitative because of the uncertainty in the determination of the spectral type from the $\mathrm{CM}$ diagram and because the fitting photosphere model extends only to $50000 \mathrm{~K}$.

The fitted effective temperatures of stars 5,10 , and 16 obtained from the SED is $\sim 6000 \mathrm{~K}$ and for star 20 is $\sim 20000 \mathrm{~K}$. These values are too low for a O- or early B-type star. For the remaining sources $8,9,12,15,17,18,20$, and 21 the fitted temperature is $\sim 50000 \mathrm{~K}$, which is in closer agreement with the effective temperature expected for an O-type star (see Schaerer $\&$ de Koter 1997). Thus, based on their position inside the bubble, we suggest that sources 8,12 , and 15 are the most reliable ionizing-star candidates of N65.

\subsection{Star formation around N65}

Figure 9 shows the distribution of both class I (yellow crosses) and class II (magenta crosses) point sources around N65. We 


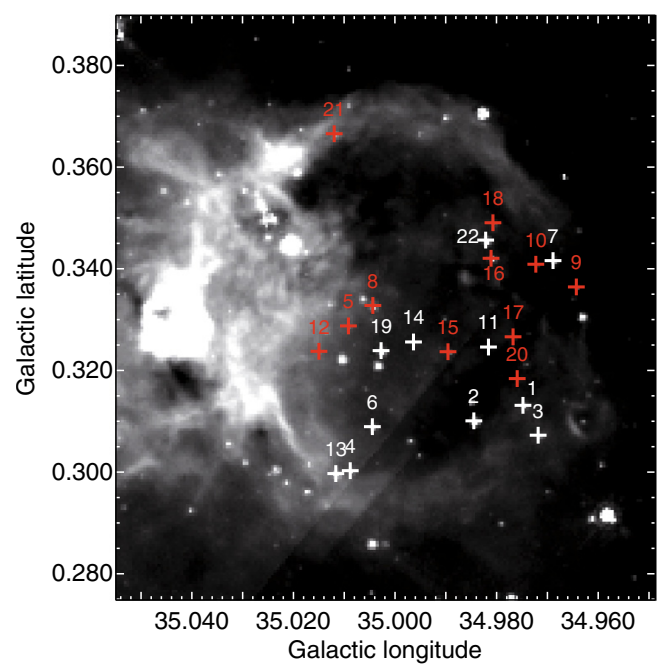

Fig. 8. Possible exciting star(s) over the $8 \mu \mathrm{m}$ emission of N65. The red crosses are the sources selected, according to the CM and the CC diagrams, to be candidate exciting stars.

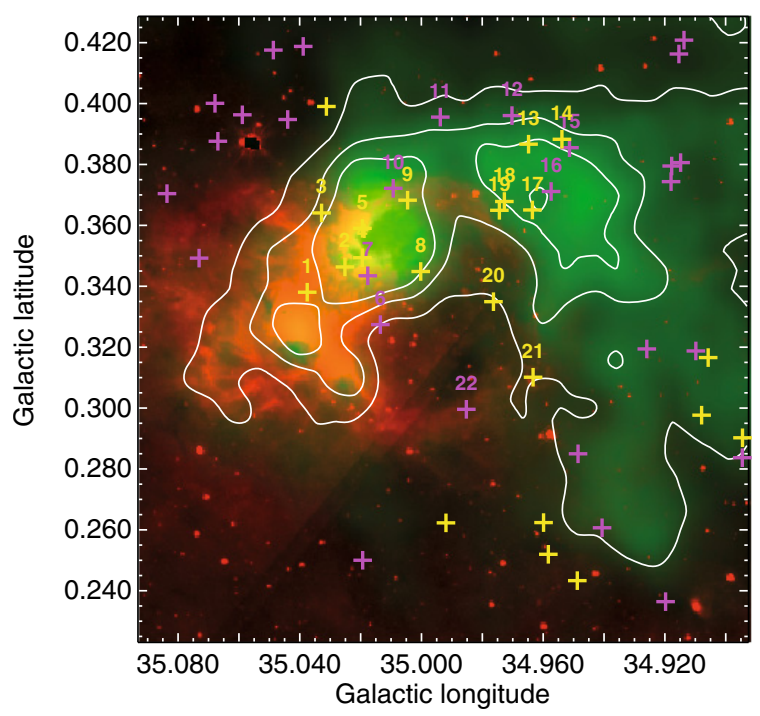

Fig. 9. Two-color image towards $\mathrm{N} 65:{ }^{13} \mathrm{CO}$ emission integrated between 47 and $55 \mathrm{~km} \mathrm{~s}^{-1}$ (in green with white contours) and $8 \mu \mathrm{m}$ emission (in red). We show the distribution of class I (yellow crosses) and class II (magenta crosses) sources around N65 derived from the CC diagram of Fig. 5. We have labeled the sources located upon the molecular cloud and close to the PDR.

note the presence of a group of these sources right upon the molecular clumps that surround the bubble above its PDR. As molecular clouds are star birth places, we expect some of these sources to be YSOs whose formation could have been triggered by the expanding IR dust bubble.

We perform a fitting of the fluxes of YSO candidates in the IRAC and 2MASS bands, to derive their spectral energy distribution (SED) and constrain their evolutionary stage. We limit our study to the sources superimposed on the molecular cloud around N65 (see Fig. 9). The SED was obtained using the tool developed by Robitaille et al. (2007) available online ${ }^{5}$. This tool consists of a grid of precomputed radiative transfer models with

5 http://caravan.astro.wisc.edu/protostars/ a fast $\chi^{2}$ minimization algorithm. We select the models that satisfied the condition

$\chi^{2}-\chi_{\min }^{2}<2 N$

where $\chi_{\min }^{2}$ is the minimum value of the $\chi^{2}$ among all models, and $N$ is the number of input data fluxes (fluxes specified as upper limit do not contribute to $N$ ). Hereafter, we refer to models satisfying Eq. (1) as "selected models".

To relate the SED to the evolutionary stage of the YSO, Robitaille et al. (2006) defined three different stages as a complementary classification of the traditional classes I, II and III. The stage classification is based on the values of the central source mass $M_{\star}$, the disk mass $M_{\text {disk }}$, and the envelope accretion rate $\dot{M}_{\text {env }}$ of the YSO. Stage I YSOs are those that have $\dot{M}_{\text {env }} / M_{\star}>$ $10^{-6} \mathrm{yr}^{-1}$, i.e., protostars with large accretion envelopes; stage II are those with $M_{\text {disk }} / M_{\star}>10^{-6}$ and $\dot{M}_{\text {env }} / M_{\star}<10^{-6} \mathrm{yr}^{-1}$, i.e., young objects with prominent disks; and stage III are those with $M_{\text {disk }} / M_{\star}<10^{-6}$ and $\dot{M}_{\text {env }} / M_{\star}<10^{-6} \mathrm{yr}^{-1}$, i.e., evolved sources where the flux is dominated by the central source. The stage classification is based on the physical properties of the YSO, rather than on the spectral index derived from the slope of its SED. However, as pointed by Robitaille et al. (2006), the stage classification is equivalent to the class classification for a great number of models. Therefore, an easy way of identifying young objects is looking for sources that can be fitted only by selected models with $\dot{M}_{\text {env }}>0$. Sources that are fitted by models with zero and non-zero values of $\dot{M}_{\text {env }}$ may be more evolved YSOs whose evolutionary stage is not constrained by the data (see Poulton et al. 2008).

In Table 1, we report the main results of the fitting output for class I and II sources projected onto the molecular cloud. The YSO identification (Col. 1) is taken from Fig. 9. In Cols. 2 and 3, we report the $\chi^{2}$ per data point of the best-fit model and the number on models satisfying Eq. (1), respectively. The remaining columns report the physical parameters of the source, specifying the range of values of the selected models: central source mass, disk mass, envelope mass, and envelope accretion rate, respectively. These results permit us to confirm the presence of young objects in the vicinity of the IR dust bubble N65.

We identify 12 sources that are only fitted by models with $\dot{M}_{\text {env }}>0$; they are sources $5,6,7,10,11,12,13,15,17$, 18,19 , and 20 . These are presumably young objects embedded in prominent envelopes. In every case, the selected models are prevalently stage I with a few stage II. The only exception is source 13, which is fitted by both stage I and stage III models.

Sources 5, 15, and 20 appear to be the youngest YSOs. They may all be massive central sources (between 8 and $10 M_{\odot}$ ) surrounded by envelopes of several solar masses. In the case of 15 and 20, their ages are estimated to be $\sim 10^{4}$ years. Regarding the source 5 , its position is coincident with the ultra-compact HII region $\mathrm{G} 35.02+0.35$ and its age is about $10^{3}$ years. This is a really young massive source and a clear sign that star formation is still occurring in the vicinity of N65.

The selected models (i.e., models satisfying Eq. (1)) for sources 7, 10, 11, and 12 spread over a similar range of values among them. These YSOs contain a central source of mass less than $5 M_{\odot}$ and ages between $10^{4}$ and $10^{5}$ years.

For source 19, we obtain a large number of selected models (85) because for this source we only fitted the four IRAC fluxes. However, all of them are stage I so this source is probably a young object. The large spread in the range of parameters does not allow us to constraint its mass and age.

Projected onto the molecular cloud, we also identify sources with zero and non-zero values for $\dot{M}_{\text {env }}$ : sources $1,2,3,4,9,14$, 
Table 1. Parameters of class I and II YSO candidates derived from the SED fitting.

\begin{tabular}{ccccccc}
\hline \hline Source & $\chi_{\min }^{2} / N$ & $n$ & $\begin{array}{c}M_{\star} \\
\left(M_{\odot}\right)\end{array}$ & $\begin{array}{c}M_{\text {disk }} \\
\left(M_{\odot}\right)\end{array}$ & $\begin{array}{c}M_{\text {env }} \\
\left(M_{\odot}\right)\end{array}$ & $\begin{array}{c}\dot{M}_{\text {env }} \\
\left(M_{\odot} / \mathrm{yr}\right)\end{array}$ \\
\hline 1 & 0.13 & 484 & $1-11$ & $8.3 \times 10^{-8}-0.4$ & $6.4 \times 10^{-9}-1800$ & $0-0.003$ \\
2 & 2.2 & 41 & $5-8$ & $7.1 \times 10^{-4}-0.7$ & $1.1 \times 10^{-8}-1200$ & $0-0.002$ \\
3 & 0.1 & 84 & $1-11$ & $1.1 \times 10^{-6}-0.71$ & $1.7 \times 10^{-8}-140$ & $0-0.001$ \\
4 & 0.2 & 273 & $0.2-11$ & $5.5 \times 10^{-5}-0.6$ & $3.8 \times 10^{-9}-360$ & $0-0.003$ \\
5 & 5 & 4 & 9 & 0 & 28 & $3.8 \times 10^{-4}$ \\
6 & 2.2 & 2 & 4.6 & 0.014 & 37 & $2.1 \times 10^{-4}$ \\
7 & 10 & 9 & $4-5$ & $7.4 \times 10^{-5}-0.02$ & $0.1-4$ & $5 \times 10^{-6}-9 \times 10^{-5}$ \\
8 & 1.3 & 1 & 9.6 & 0.75 & 130 & $2.1 \times 10^{-4}$ \\
9 & 5 & 61 & $2-7$ & $1.6 \times 10^{-7}-0.13$ & $1.8 \times 10^{-7}-17$ & $0-5.4 \times 10^{-4}$ \\
10 & 4 & 9 & 5 & $4.5 \times 10^{-5}-0.001$ & $0.09-1.5$ & $9.5 \times 10^{-7}-1.4 \times 10^{-5}$ \\
11 & 3.3 & 10 & $2-4$ & $6.5 \times 10^{-4}-0.03$ & $0.02-16$ & $4 \times 10^{-6}-5 \times 10^{-4}$ \\
12 & 11 & 2 & $3-4$ & $0.016-0.02$ & $2-8$ & $3.5 \times 10^{-6}-2 \times 10^{-5}$ \\
13 & 2.4 & 13 & $1-7$ & $1.1 \times 10^{-6}-0.3$ & $0.006-43$ & $3.5 \times 10^{-8}-1.1 \times 10^{-4}$ \\
14 & 1.4 & 298 & $1-11$ & $3 \times 10^{-6}-0.7$ & $5 \times 10^{-9}-140$ & $0-0.002$ \\
15 & 1 & 3 & $8-9$ & $0.03-0.2$ & $70-140$ & $1.4 \times 10^{-8}-2 \times 10^{-4}$ \\
16 & 0.03 & 430 & $1-8$ & $3 \times 10^{-8}-0.4$ & $1 \times 10^{-8}-23$ & $0-4 \times 10^{-4}$ \\
17 & 3 & 2 & 4.6 & 0.014 & 37 & $2.1 \times 10^{-4}$ \\
18 & 0.8 & 13 & $2-9$ & $6.7 \times 10^{-4}-0.7$ & $0.1-140$ & $3 \times 10^{-6}-4 \times 10^{-4}$ \\
19 & 0.4 & 85 & $2-21$ & $0-1.1$ & $0.4-1300$ & $4.1 \times 10^{-5}-0.002$ \\
20 & 2 & 2 & $9-10$ & $0.3-0.8$ & $65-130$ & $2 \times 10^{-4}$ \\
21 & 0.003 & 1652 & $0.7-15$ & $6 \times 10^{-7}-0.9$ & $4.5 \times 10^{-9}-560$ & $0-0.001$ \\
22 & 1.3 & 50 & $3-15$ & $9 \times 10^{-9}-0.2$ & $1.4 \times 10^{-8}-200$ & $0-6 \times 10^{-4}$ \\
\hline
\end{tabular}

16,21 , and 22 . The evolutionary stage of these sources cannot be assured without doubt because the parameters of the selected models are spread over a wide range. We should include fluxes measured at other wavelengths to reduce the number of models satisfying the condition defined in Eq. (1). However, we can identify sources that are in their early stages of evolution (embedded or disk dominated) because the selected models are either stage I or II. This is the case for sources 1, 2, 4, 14, and 16. For the remaining sources, $3,9,16$, and 22, the selected models are distributed between stages I, II, and III so their evolutionary stage cannot be established without controversy.

We note that source 21 is located at the center of the smaller IR bubble introduced in Sect. 3 that lies right upon the PDR of N65 (see Fig. 2). We fitted the SED of the source and obtained more than 1600 models satisfying Eq. (1) and, consequently, a wide range of physical parameters for the YSO, so we cannot establish its evolutionary stage. However, its location in the center of the smaller IR dust bubbles suggests that this source may be a second generation massive star blowing a stellar wind strong enough to evacuate a bubble around itself. More observations are needed to investigate this scenario.

In conclusion, the SED study confirms the existence of several YSOs possibly embedded in the molecular clumps that surround N65, which is indicative of the collect and collapse process occurring in this region.

\subsection{SED analysis of the EGO G35.03+0.35}

We derive the SED for the EGO G35.05+0.35 using the fluxes in the Spitzer-IRAC 4.5, 5.8, and $8.0 \mu \mathrm{m}$ bands (the GPSC reports no detection in the $3.6 \mu \mathrm{m}$ band and the source is absent in the 2MASS catalog). We also use fluxes in the far IR of the SCUBA bands of 450 and $850 \mu \mathrm{m}$ and in the band of $1200 \mu \mathrm{m}$ measured by the SIMBA bolometer at SEST (Hill et al. 2006). Both the SCUBA and SIMBA datasets have a lower angular resolution than that of the GPSC and the measured fluxes may

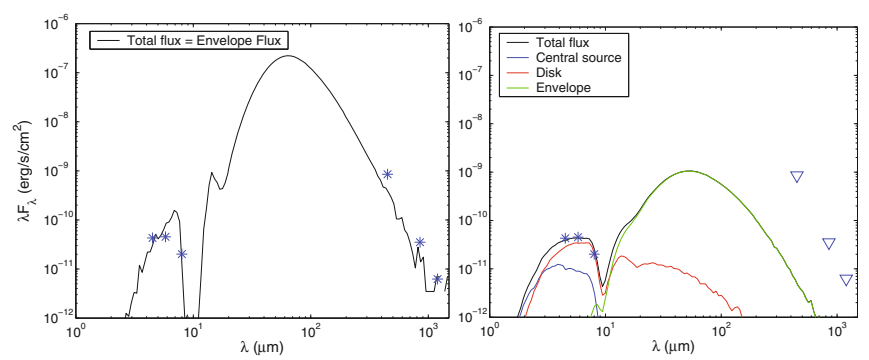

Fig. 10. Best-fit SED model for the EGO G35.05+0.35. The points in blue are the measured fluxes at the Spitzer-IRAC bands of 4.5, 5.8, and $8.0 \mu \mathrm{m}$, the SCUBA bands of 450 and $850 \mu \mathrm{m}$, and the band of $1,200 \mu \mathrm{m}$ measured with the SIMBA bolometer at SEST. Left: SCUBA and SIMBA data are assumed to represent flux emitted only by the EGO. The total flux (black line) is completely dominated by the flux from the envelope as would be expected in a young stellar object at its earlier stages of evolution. Right: SCUBA and SIMBA data are taken to be an upper limit. The total flux at the longer wavelengths is dominated by the envelope, while at the shorter wavelengths the main contributions originates in the disk (red line) and the central source (blue line).

include contributions from other sources near the EGO. For this reason, we perform a fitting of the SED by considering two different cases: a) assuming that the far IR data represent the flux emitted only by the EGO, the SED of the best-fit model being shown in Fig. 10 (left); and b) using the far IR data as an upper limit (i.e., assuming that the emission originates in the EGO and, probably, other sources around it), the SED of the best-fit model in this case being shown in Fig. 10 (right). In Table 2, we report the physical parameters of the selected models, considering both SCUBA and SIMBA data as flux emitted only by the EGO (case $a$ ) and upper limits (case $b$ ). The column designation is the same as in Table 1.

According to Fig. 10 (left), if we consider fluxes at the longer wavelengths to be emitted only by the EGO, the total flux of the best-fit model is completely dominated by the envelope. As 
Table 2. Physical parameters of EGO G35.05+0.35 derived from the SED fitting for the selected models satisfying Eq. (1).

\begin{tabular}{ccccccc}
\hline \hline Case & $\chi_{\min }^{2} / N$ & $n$ & $\begin{array}{c}M_{\star} \\
\left(M_{\odot}\right)\end{array}$ & $\begin{array}{c}M_{\text {disk }} \\
\left(M_{\odot}\right)\end{array}$ & $\begin{array}{c}M_{\text {env }} \\
\left(M_{\odot}\right)\end{array}$ & $\begin{array}{c}\dot{M}_{\text {env }} \\
\left(M_{\odot} / \mathrm{yr}\right)\end{array}$ \\
\hline $\mathrm{a}$ & 30 & 2 & $27-33$ & $0-0.3$ & $1900-2500$ & $0.004-0.006$ \\
$\mathrm{~b}$ & 3.3 & 16 & $6-10$ & $0.005-0.8$ & $0.7-1100$ & $2 \times 10^{-5}-0.002$ \\
\hline
\end{tabular}

pointed out by Robitaille et al. (2006), the envelope of sources with high accretion rates becomes optically thick. In our case of $\dot{M}_{\text {env }} \sim 10^{-3} M_{\odot} / \mathrm{yr}$, radiation emitted by both the central source and the disk is probably absorbed and re-emitted by the envelope of gas and dust. As can be seen in Table 2, the physical parameters of the EGO indicate that it is a massive central source surrounded by a massive envelope. According to Fig. 10 (right), if we take SCUBA and SIMBA measurements to be upper limits, the total flux of the best-fit model is still dominated by the envelope but only at the longer wavelengths. In the near and mid IR, the main contribution to the total flux comes from the disk. The accretion rate values that we derived for the envelope are lower than those derived in the previous case (see Table 2). Thus, the envelope is expected to be more transparent to radiation coming from the inner regions (disk and central source).

Both cases show that the fitted SED of the EGO is indicative of a massive central source surrounded by a massive envelope. The age of the YSO is estimated in-between $10^{4}$ and $10^{5}$ years. From these results, we suggest that the EGO G35.05+0.35 is a candidate massive young stellar object at the earlier stages of evolution with outflowing activity, in agreement with that proposed by Cyganowski et al. (2008). The presence of this object is a clear sign that star formation is occurring in the vicinity of N65.

\section{Summary}

We have conducted a study of the surroundings of the IR dust bubble N65 using large-scale surveys and archival data in the radio and infrared bands. The main results can be summarized as follows:

1. We discovered the presence of a molecular cloud with an arc-like shape enclosing N65 over its east, north and west borders and centered on a velocity of $\sim 50 \mathrm{~km} \mathrm{~s}^{-1}$. The cloud appears to be fragmented into smaller molecular clumps that are seen right upon the PDR. Based on the distribution and morphology of the molecular gas between 47 and $55 \mathrm{~km} \mathrm{~s}^{-1}$, we suggest that the collect and collapse process may be occurring.

2. The EGO G35.05+0.35 lies in the most intense molecular clump, which belongs to the discovered molecular shell. From the ${ }^{13} \mathrm{CO} J=1-0$ emission, we estimated its molecular mass and density to be $\sim 2 \times 10^{3} M_{\odot}$ and $\sim 10^{4} \mathrm{~cm}^{-3}$, respectively. We found that the dust continuum emission, traced by the $850 \mu \mathrm{m}$ band, also peaks in this region. We estimated a dust-to-gas ratio of $\sim 0.003$ for this clump.

3. From the analysis of the infrared photometry of point sources around N65, we identified three O- or early B-type stars as the most probable candidates responsible for the creation of N65. In addition, we found many YSOs distributed right upon the molecular clumps around N65. The analysis of the SED of these sources confirms that many of them are in the embedded or disk-dominated stage. This suggests that the gas collected into the molecular clumps may have collapsed to form a second generation of stars, showing another galactic case of the collect and collapse process.

4. The SED of EGO G35.05+0.35 as derived by fitting the fluxes at the mid and far IR, confirms that it is a massive young stellar object.

Acknowledgements. A.P. is a doctoral fellow of CONICET, Argentina. S.P. and E.G. are members of the Carrera del investigador cientifico of CONICET, Argentina. This work was partially supported by the CONICET grant PIP 112200801-02166, UBACYT A023 and ANPCYT PICT-2007-00902.

\section{References}

Allen, L. E., Calvet, N., D’Alessio, P., et al. 2004, ApJS, 154, 363 Anderson, L. D., \& Bania, T. M. 2009, ApJ, 690, 706

Anderson, L. D., Bania, T. M., Jackson, J. M., et al. 2009, ApJS, 181, 255

Bessell, M. S., \& Brett, J. M. 1988, PASP, 100, 1134

Bronfman, L., Nyman, L.-A., \& May, J. 1996, A\&AS, 115, 81

Caswell, J. L., Vaile, R. A., Ellingsen, S. P., Whiteoak, J. B., \& Norris, R. P. 1995, MNRAS, 272, 96

Churchwell, E., Povich, M. S., Allen, D., et al. 2006, ApJ, 649, 759

Churchwell, E., Watson, D. F., Povich, M. S., et al. 2007, ApJ, 670, 428

Cyganowski, C. J., Whitney, B. A., Holden, E., et al. 2008, AJ, 136, 2391

Deharveng, L., Zavagno, A., \& Caplan, J. 2005, A\&A, 433, 565

Di Francesco, J., Johnstone, D., Kirk, H., MacKenzie, T., \& Ledwosinska, E. 2008, ApJS, 175, 277

Elmegreen, B. G., \& Lada, C. J. 1977, ApJ, 214, 725

Fazio, G. G., Hora, J. L., Allen, L. E., et al. 2004, ApJS, 154, 10

Fich, M., Blitz, L., \& Stark, A. A. 1989, ApJ, 342, 272

Forster, J. R., \& Caswell, J. L. 1989, A\&A, 213, 339

Hill, T., Thompson, M. A., Burton, M. G., et al. 2006, MNRAS, 368, 1223

Jackson, J. M., Rathborne, J. M., Shah, R. Y., et al. 2006, ApJS, 163, 145

Jijina, J., Myers, P. C., \& Adams, F. C. 1999, ApJS, 125, 161

Pomarès, M., Zavagno, A., Deharveng, L., et al. 2009, A\&A, 494, 987

Poulton, C. J., Robitaille, T. P., Greaves, J. S., et al. 2008, MNRAS, 384, 1249

Rieke, G. H., \& Lebofsky, M. J. 1985, ApJ, 288, 618

Robitaille, T. P., Whitney, B. A., Indebetouw, R., Wood, K., \& Denzmore, P. 2006, ApJS, 167, 256

Robitaille, T. P., Whitney, B. A., Indebetouw, R., \& Wood, K. 2007, ApJS, 169, 328

Schaerer, D., \& de Koter, A. 1997, A\&A, 322, 598

Simon, R., Jackson, J. M., Clemens, D. P., Bania, T. M., \& Heyer, M. H. 2001, ApJ, 551, 747

Tej, A., Ojha, D. K., Ghosh, S. K., et al. 2006, A\&A, 452, 203

Watson, C., Araya, E., Sewilo, M., et al. 2003, ApJ, 587, 714

Werner, M. W., Roellig, T. L., Low, F. J., et al. 2004, ApJS, 154, 1

Zavagno, A., Pomarès, M., Deharveng, L., et al. 2007, A\&A, 472, 835 